\title{
REIATIONSHIPS BETWEEN CLIMATIC VARIATION \\ AND GRASSLAND BIOMASS ON THE NORTHERN RANGE OF YELIOWSTONE NATIONAL PARK
}

\author{
Mark S. Boyce \\ Evelyn H. Merrill \\ Department of Zoology and Physiology \\ and \\ Ronald Marrs \\ Department of Geology \\ University of Wyoming \\ Laramie
}

\section{Objectives}

Summer range is not thought to be limiting to elk because abundant, high quality forage is available whereas a shortage of forage usually exists on elk winter ranges. Nevertheless, summer nutrition and fat reserves acquired on summer ranges influence growth, survival and reproduction of ungulates (Klein 1965, Verme 1963, 1965, Julander et al. 1961). The enormous area of summer range for the Northern Yellowstone elk herd renders detailed field surveys impractical. This study was initiated to evaluate the feasibility of using LANDSAT satellite imagery to (1) characterize vegetation communities on summer range from LANDSAT multispectral scanner (MSS) data and digitized vegetation maps of Yellowstone National Park, (2) predict plant production within grassland types and (3) correlate elk population characteristics with fluctuations in plant production and weather.

\section{Methods}

Computer compatible tapes (CCT) for LANDSAT MSS data for 9 years within the period 1972-1986 were obtained and catalogued into the University of Wyoming's Cyber Computer Library. The 1987 LANDSAT imagery of the Park was taken on 5 August during a period of less than $30 \%$ cloud cover. Computer programs were written to dump the LANDSAT MSS data in interleaved and sequenced band EDIPS format from the CCT to a format suitable to use in the LANDSAT Multi-Image Processing System (MIPS).

\section{Vegetation classification}

Classification of major habitat types on elk summer range using LANDSAT MSS data is in progress. Co-registration of digitized Yellowstone National Park habitat maps and LANDSAT MSS data using the MIPS image processing system has proved impractical and a supervised classification approach using the original vegetation maps is being pursued. Reflectance values from a total of 200 pixels within vegetatively 
homogeneous sites, of not less than 20 ha in size, have been identified on Yellowstone National Park vegetation maps. Spectral signatures for these training sites are being determined from 1972 LANDSAT MSS data which has been smoothed using a low-pass filter ( $3 \times 3$ pixels). These data will be subjected to discriminant analysis to obtain vegetation classification functions.

\section{Grassland production}

The feasibility of predicting vegetation production within grasslands from LANDSAT MSS data is being determined using 1987 LANDSAT spectral information and ground vegetation sampling. Vegetative production during 1987 was sampled from 9-2l August at 24 sites ( $<12$ ha) on 3 major elk summer ranges (Mirror Plateau, Norris/Cache-Calfee Ridge Complex, Buffalo Plateau) and at 2 high elevational sites on Mt. Washburn. Sites were classified as to physiographic features and habitat types based on habitat maps and descriptions received from the Park. Green biomass of 3 forage classes (forbs, graminoids and shrub current annual growth) were estimated using a capacitance meter or, in areas into which the capacitance meter could not be transported, from indices of plant volume (canopy coverage $x$ average height). A total of 270 microplots were clipped to derive regression models for predicting green plant biomass from capacitance readings and/or volume indices. Clipped plant material was separated into green biomass by forage class and litter, oven dried at 100 degrees $C$ for 24 hours and weighed to the nearest 0.01 gram.

Spectral reflectance values of the 4 MSS bands at the 26 sites sampled were identified in the 1987 LANDSAT data by co-registering a portion of the 1987 LANDSAT scene and IR photos on which the 26 sites were mapped. Reflectance signatures of the 26 sites have been recorded.

\section{Results}

\section{Vegetation classification}

Classification analysis of major habitat types based on LANDSAT imagery has not yet been completed. However, a procedure for delineating timbered areas from grassland/sagebrush-grassland sites has been developed based on principal components analysis. This procedure has been used to create a vegetation "mask" designed to identify and extract LANDSAT spectral information only from grassland/sagebrush-grassland areas designated as elk summer range. This "mask" will be used on LANDSAT imagery for the period 1972-1987 for predicting total grassland production on elk summer ranges during these years.

\section{Grassland production}

Twenty-one grassland and 5 sites dominated by sagebrush-grassland were sampled during August 1987 (Table 1). This is less than half the number of sample sites we had originally desired. However, logistical 
Table 1. Sum mary of site characteristics where plant production was sampled during August 1987.

\begin{tabular}{|c|c|c|c|c|c|c|}
\hline Plot \# & $\begin{array}{l}\text { Date } \\
\text { Sampled }\end{array}$ & $\begin{array}{l}\text { Plot } \\
\text { Location }\end{array}$ & $\begin{array}{l}\text { abitat } \\
\text { ype }\end{array}$ & $\begin{array}{l}\text { Elevation } \\
\text { (feet) }\end{array}$ & $\begin{array}{l}\text { Aspect } \\
\text { (degrees) }\end{array}$ & $\begin{array}{l}\text { Slope } \\
\text { (908) }\end{array}$ \\
\hline 1 & $8 / 9 / 87$ & Mt. Nonis & FN & 7840 & 260 & 20 \\
\hline 2 & $8 / 9 / 87$ & Mt. Norris & TFG & 8000 & 185 & 13 \\
\hline 3 & $8 / 10 / 87$ & Mt. Nonris & FNG & 7600 & 200 & 10 \\
\hline 4 & $8 / 10 / 87$ & Mt. Norris & $\mathrm{TA}$ & 8200 & 190 & 32 \\
\hline 5 & $8 / 10 / 87$ & Mt Nonis & FNG & 8200 & 110 & 33 \\
\hline 6 & $8 / 11 / 87$ & Mt Washburn & FA & 8740 & 240 & 37 \\
\hline 7 & $8 / 11 / 87$ & Mt. Washburn & FA & 8700 & 280 & 22 \\
\hline 8 & $8 / 12 / 87$ & Opal Creek & DW & 8750 & 312 & 15 \\
\hline 9 & $8 / 13 / 87$ & Opal Creek & DW & 9060 & Flat & 0 \\
\hline 10 & $8 / 13 / 87$ & Opal Creek & FD & 9000 & 103 & 18 \\
\hline 11 & $8 / 13 / 87$ & Opal Creed & FD & 8900 & 150 & 23 \\
\hline 12 & $8 / 13 / 87$ & Opal Creek & DC & 8800 & Flat & 4 \\
\hline 13 & $8 / 14 / 87$ & $\begin{array}{l}\text { Specimen } \\
\text { Ridge Trail }\end{array}$ & FN & 9150 & 200 & 21 \\
\hline 14 & $8 / 14 / 87$ & $\begin{array}{l}\text { Specimen } \\
\text { Ridge }\end{array}$ & FD & 9150 & 90 & 5 \\
\hline 15 & $8 / 14 / 87$ & $\begin{array}{l}\text { Specimen } \\
\text { Ridge }\end{array}$ & FNG & 8800 & 120 & 30 \\
\hline 16 & $8 / 16 / 87$ & $\begin{array}{l}\text { Buffalo } \\
\text { Plateau }\end{array}$ & FA & 6400 & 250 & 5 \\
\hline 17 & $8 / 16 / 87$ & $\begin{array}{l}\text { Buffalo } \\
\text { Plateau }\end{array}$ & $\mathrm{TF}$ & 6700 & 240 & 20 \\
\hline 18 & $8 / 17 / 87$ & $\begin{array}{l}\text { 2B-1 Camp- } \\
\text { Ground }\end{array}$ & FNG & 8900 & 180 & 15 \\
\hline 19 & $8 / 17 / 87$ & $\begin{array}{l}\text { Little Buffalo } \\
\text { Creek }\end{array}$ & FD & 8820 & 180 & 3 \\
\hline 20 & $8 / 19 / 87$ & Soda Butte & $\mathrm{TF}$ & 6620 & Flat & 0 \\
\hline 21 & $8 / 19 / 87$ & $\begin{array}{l}\text { Lower Lamar. } \\
\text { Trail }\end{array}$ & FA & 6640 & Flat & 0 \\
\hline 22 & $8 / 19 / 87$ & $\begin{array}{l}\text { Lower Mt. } \\
\text { Nonis/ } \\
\text { Lamar Trail }\end{array}$ & FNG & 6690 & 277 & 7 \\
\hline 23 & $8 / 20 / 87$ & Cache-Calfee & FN & 8120 & 312 & 8 \\
\hline 24 & $8 / 21 / 87$ & Cache-Calfee & FN & 7920 & 267 & 4 \\
\hline 25 & $8 / 21 / 87$ & Cache Calfee & FN & 8200 & 277 & 8 \\
\hline 26 & $8 / 21 / 87$ & $\begin{array}{l}\text { East Wabb } \\
\text { Springs }\end{array}$ & FNG & 7240 & 317 & 6 \\
\hline
\end{tabular}


constraints prevented more extensive sampling in the time frame dictated by phenological change in the vegetation. Capacitance meter readings provided more precise estimates of green biomass than did visual estimates of plant volume (Table 2 ).

Total plant production ranged from 527 to $3154 \mathrm{~kg} / \mathrm{ha}$ on grassland sites and 482-1978 kg/ha on sagebrush-grassland sites (Table 3). Graminoids comprised more than $60 \%$ of the total vegetative production on grasslands, except on the Buffalo Plateau in the area above campground $2 \mathrm{~B}-1$. Forbs comprised more than $70 \%$ of the plant biomass at this site (Table 3). Current annual growth of sagebrush comprised 18-40\% of total plant production on the sagebrush-grassland sites.

At present, we have compared estimates of grassland production with 3 vegetation indices based on spectral ratios which are commonly used to enhance variations in the vegetation component of LANDSAT spectral data. These indices include the normalized difference (ND), leaf area index (LAI) and infra-red/red band ratio (IR/RED) (Curran 1983, Crist and Cicone 1984, Crist and Kauth 1986). Correlations among these vegetation indices and field estimates of total plant biomass on all sites have been weak with the strongest correlations observed between vegetative indices and forb biomass. Further development of algorithms for predicting total plant production is in progress.

Climatic data

Weather data for the years 1972-1986 have been compiled and computerized for 3 weather stations.

Elk population characteristics

Data analysis on elk population characteristics has been initiated but is not yet complete. We anticipate further data compilation and analysis after a trip to Yellowstone National Park in early February.

\section{Conclusions}

Since data analysis is still in progress, it is inappropriate to suggest conclusions at this time. Initial correlations between total green biomass on all sites and vegetation indices derived from LANDSAT imagery have been weak and may reflect differences in phenology since increases in photosynthesis will increase spectral reflectance regardless of the amount of biomass (Carneggie et al. 1974). However, additional analysis is in progress. 


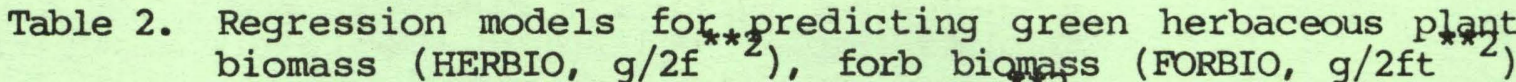
graminoid biomass (GRASSBIO, g/2ft ${ }^{2}$ ) and current annual growth of shrubs ( $C A G, g / 2 \mathrm{ft}^{* 2}$ ) from capacitance meter readings [shrub, (SHCAP) and herbeceous plant (HCAP) compacitance reading], and plant volume indices (FORBVOL, GRASSVOL) on sites sampled in Yellowstone National Park. The natural logarithm is denoted by $\mathrm{ln}$.

\begin{tabular}{lllll}
\hline \multicolumn{1}{c}{$Y$} & Regression Model & S.E. & $\mathrm{R}^{\star \star 2}$ & $\mathrm{~N}$ \\
\hline In CAG & $=0.062+1.081$ SHCAP & 0.439 & 0.86 & 143 \\
HERBIO & $=4.262+0.598$ HCAP & 8.607 & 0.73 & 133 \\
FORBIO & $=2.509+0.014$ FORBVOL & 3.223 & 0.453 & 202 \\
GRASSBIO & $=4.504+0.011$ GRASSVOL & 9.006 & 0.626 & 240 \\
\hline
\end{tabular}


Table 3. Green biomass ( $\mathrm{kg} / \mathrm{ha}$ ) and bareground canopy coverage $(\xi)$ at 26 plots sampled 9-2l August 1987 on elk summer range in Yellowstone National Park.

\begin{tabular}{rrrrrrr}
\hline $\begin{array}{c}\text { Plot } \\
\text { Number }\end{array}$ & $\begin{array}{c}\text { Total } \\
\text { Biomass }\end{array}$ & $\begin{array}{c}\text { Total } \\
\text { Herb }\end{array}$ & Browse & Forb & Grass & $\begin{array}{c}\text { Bare- } \\
\text { Ground }\end{array}$ \\
\hline 1 & 616.7 & 580.2 & 36.5 & 167.1 & 413.1 & 22.2 \\
2 & 1978.7 & 1193.6 & 785.1 & 366.1 & 827.5 & 10.6 \\
3 & 3153.9 & 3153.9 & 0 & 297.8 & 2856.2 & 3.3 \\
4 & 885 & 638.7 & 246.3 & 183.7 & 455 & 34.4 \\
5 & 960.2 & 960.2 & 0 & 267.6 & 692.6 & 31.7 \\
6 & 673.4 & 638.1 & 35.3 & 203.5 & 434.6 & 2.6 \\
7 & 1032.2 & 1032.2 & 0 & 138.9 & 893.3 & 2.1 \\
8 & 1077.4 & 1077.4 & 0 & 269.6 & 807.8 & 3.9 \\
9 & 694.8 & 694.8 & 0 & 178.5 & 516.3 & 7.1 \\
10 & 1087.3 & 1087.3 & 0 & 323.7 & 763.6 & 6 \\
11 & 838.6 & 838.6 & 0 & 276.6 & 561.7 & 12 \\
12 & 1151.6 & 1151.6 & 0 & 257 & 894.7 & 6 \\
13 & 1536.8 & 1536.8 & 0 & 371 & 1165.9 & 2.4 \\
14 & 1020.3 & 1020.3 & 0 & 249.8 & 770.5 & 2.9 \\
15 & 1627.9 & 1627.9 & 0 & 481.5 & 1146 & 6.3 \\
16 & 576.8 & 530.8 & 46.2 & 60.8 & 469.9 & 46.5 \\
17 & 1180.8 & 964.7 & 216.1 & 224.4 & 740.3 & 11.9 \\
18 & 907.5 & 907.5 & 0 & 714 & 193.2 & 47.7 \\
19 & 946 & 946 & 0 & 260.9 & 685.1 & 3 \\
20 & 481.9 & 363.5 & 118.4 & 80.2 & 283.3 & 6.4 \\
21 & 1030.5 & 963.4 & 67.2 & 143.3 & 820.1 & 1.6 \\
22 & 1293.3 & 896.5 & 396.8 & 142.6 & 753.9 & 2.7 \\
23 & 684.9 & 684.9 & 0 & 212.2 & 472.7 & 6.2 \\
24 & 526.5 & 526.5 & 0 & 202.3 & 324.2 & 2.6 \\
25 & 778.5 & 778.5 & 0 & 236.8 & 541.7 & 4.5 \\
26 & 1246.5 & 1246.5 & 0 & 282.2 & 964.3 & 11.9 \\
& & & & & & \\
\hline
\end{tabular}




\section{Literature Cited}

Curran, P. J. 1983. Estimating leaf area index. Photogramic Engineering and Remote Sensing. 40(12):1709.

Crist, E. P. and Kauth, R. J. 1986. The tasseled cap demystified . Photogramic Engineering and Remote Sensing 52(1):81.

Crist, E. P. and Cicone. 1984. The tasseled cap and Thematic mapper. Photogramic Engineering and Remote Sensing 50(3):343.

Julander, O., W. L. Robinette and D. A. Jones. 1961. Relation of summer range condition to mule deer herd productivity. J. Wildl. Manage. 25:54-60.

Klein, D. R. 1965. Ecology of deer range in Alaska. Ecol. Monogr. $35: 259-284$.

Verme, L. J. 1965. Reproduction studies on penned white-tailed deer fawns. J. Wildl. Manage. 29:74-79.

Verme, L. J. 1963. Effect of nutrition on growth of white-tailed deer fawns. Trans. N. Am. Wildl. Nat. Res. Conf. 28:431-443. 